\title{
Introduction au dossier
}

\section{Laurence Boutinot}

\section{(2) OpenEdition}

1 Journals

Édition électronique

URL : http://journals.openedition.org/anthropodev/466

DOI : 10.4000/anthropodev.466

ISSN : 2553-1719

Éditeur

APAD - Association pour l'anthropologie du changement social et du développement

\section{Édition imprimée}

Date de publication : 1 avril 2014

Pagination : 29-42

ISBN : 979-10-93476-00-1

ISSN : 2276-2019

\section{Référence électronique}

Laurence Boutinot, «Introduction au dossier », Anthropologie \& développement [En ligne], 37-38-39 | 2014, mis en ligne le 01 décembre 2016, consulté le 03 mai 2019. URL : http:// journals.openedition.org/anthropodev/466 ; DOI : 10.4000/anthropodev.466

\section{(c) (1)}

La revue Anthropologie \& développement est mise à disposition selon les termes de la Licence Creative Commons Attribution 4.0 International. 


\title{
Acteurs et réformes de la gestion des ressources forestières en Afrique de l'Ouest
}

\author{
Introduction au dossier
}

\section{Laurence Boutinot}

La gestion des ressources naturelles en Afrique, la décentralisation et les réformes des services publics ont fait l'objet de travaux publiés dans un certain nombre de numéros précédents du Bulletin de l'APAD depuis une vingtaine d'années. Ce numéro de la nouvelle Revue Anthropologie et développement voudrait rendre hommage au professeur Bréhima Kassibo, ami et collègue, qui a participé activement à la vie de l'association pour l'anthropologie du développement et du changement social (APAD), à ses manifestations, à ses colloques successifs, à ses publications et qui nous a quitté récemment. En 1997, dans le Bulletin de l'APAD n 14, Bréhima Kassibo (ed, 1997) ouvrait la voie à l'étude de la décentralisation dans le nouveau contexte du multiparitisme au Mali, qui suscitait beaucoup d'espoirs démocratiques. En 1998, Giorgio Blundo et Roch Mongbo (eds, 1998) écrivaient dans le numéro suivant, que la décentralisation comme enjeu concret, comme enjeu politique et comme objet d'étude, avait « de beaux jours devant elle ». Ces dossiers ont non seulement ouvert une série de recherches sur la délivrance des services publics, ils ont également été à l'initiative des critiques des conceptions normatives que véhiculait la notion de "bonne gouvernance", telle que promue par les institutions d'aide au développement.

Le présent dossier entend poursuivre ces réflexions sur le terrain spécifique des réformes en matière de gestion des ressources forestières en Afrique de l'Ouest - le Mali, le Sénégal et le Niger en particulier- dans la première décennie de ce XXle siècle - à travers la description et l'analyse des processus de décentralisation et de négociations entre les différents acteurs stratégiques du développement local et national. Ce dossier est réalisé dans le contexte des réformes de l'action publique que traduisent les politiques de décentralisation, la délégation de compétences aux ac- 
teurs locaux, qui vont de pair avec l'expérimentation de formes nouvelles de participation et d'empowerment des populations locales. De même, il voudrait questionner les ambiguïtés de ces réformes et les tensions dans l'usage du concept de gouvernance et de ses avatars : transparence, accountability. En effet, l'opérationnalité de ces concepts pour traiter de/ou assurer concrètement une gestion durable des ressources et des terres n'est pas avérée.

\section{La spécificité de la gestion des ressources forestières}

Les ressources forestières sont des objets qui ne relèvent pas du seul souci écologique ou d'un traitement technique. Elles sont au cœur des rapports sociaux, économiques et politiques inscrits dans l'histoire longue des territoires et des hommes. Leur gestion a cela de particulier qu'elle donne à voir les divergences d'intérêts, les différentes logiques coutumières, administratives ou techniciennes, et oblige à prendre au sérieux les fondements sociaux des structures de pouvoir et leurs légitimités sur les espaces, les terres et les territoires. La délégation de compétences de la gestion foncière et forestière aux collectivités locales est contenue dans les réformes institutionnelles. Elle s'illustre de manière tout à fait spécifique dans le langage ambigu des projets de développement qui oscille entre la valorisation des biens communs, de la tradition et de la solidarité communautaire d'un côté, et la mise en œuvre d'un pluralisme institutionnel associé à une économie libérale de l'offre de biens et de services, de l'autre. Dans les projets de gestion forestière, on observe dans bien des cas que la "participation » des acteurs locaux est un exercice laborieux, souvent plus contraint que librement consenti, aussi bien du point de vue de ceux à qui il s'adresse que de celui des agents de développement. Et on est en droit de se demander si, dans certaines de ces circonstances précisément liées à la gestion des espaces et des ressources naturelles, cette participation ne tend pas à masquer la fonction déléguée aux villageois d'entretenir à moindre frais la ressource pour une exploitation durable (Kassibo, 1997 ; Boutinot, 2006).

C'est en partie dans cette ambiguïté des réformes que les concepts normatifs de bonne gouvernance s'imposent tels des modèles de gestion. Ils nécessitent la mise en place de cadres de négociation entre une multi- 
plicité d'acteurs et d'intérêts mais dont on ne sait plus trop bien si cette multiplicité est le fruit des dispositifs mis en place ou bien la cause des conflits sur les ressources. Les deux, sans doute, tant il est vrai que les formes de compétitions et les risques de surexploitation des ressources naturelles se multiplient dans le contexte de la marchandisation des biens naturels et des enjeux de la commercialisation des produits issus de la forêt.

Objets de prélèvements et non d'investissement en travail (Lavigne Delville et Hochet, 2005), les ressources naturelles renouvelables ont la spécificité d'être à la fois des biens souvent rares et des biens communs qui engagent la responsabilité de tous. Quand ces ressources font l'objet de valorisation économique, et nous le voyons bien dans les exemples présentés dans ce dossier, cela engendre une concurrence de plus en plus forte sur les territoires en termes d'accès et de contrôle. Mais dès lors qu'elles demandent un travail de transformation et deviennent source de haute valeur ajoutée comme c'est le cas pour la production du charbon de bois, ce sont aussi, ainsi que le rappelle Ribot (2003, cité par Lavigne Deville 2005 : 4), les enjeux sur le contrôle des filières de commercialisation qui font l'objet d'investissement et de luttes entre les acteurs, tel qu'on peut le voir au Mali et au Sénégal.

Les contributions de ce dossier analysent ainsi les enjeux d'appropriation, d'accès et de contrôle des espaces forestiers dans la dynamique de la décentralisation et des réformes politiques liées à la gestion de l'environnement. Le contrôle étatique centralisé sur les ressources et sur la gestion foncière, hérité de l'époque coloniale, a longtemps relégué les villageois dans un statut précaire d'usufruitiers qui subissaient le monopole de la violence légitime détenu par les services des Eaux et Forêts. Ainsi que l'écrivait Bréhima Kassibo, la décentralisation au Mali a suscité de grands espoirs pour les populations villageoises : elles avaient espéré sentir se desserrer l'étau d'un Etat autoritaire. Les enjeux principaux autour de la réappropriation par les acteurs d'une certaine agencéité dans la gestion des affaires publiques locales s'illustrent dans les articles réunis dans ce dossier, dans la possibilité de capter des revenus de l'exploitation locale du bois, d'une part, et de contrôler l'accès au territoire et aux ressources forestières, d'autre part. 


\section{Des enjeux et des conflits pour la redéfinition des territoires et des identités}

Les textes de Faty Mbodj et de Seydou Keïta traitent des forêts du Mandé, proche de la capitale malienne. Faty Mbodj présente des conflits villageois dont l'enjeu est de contrôler l'accès à la ressource et la redistribution des ristournes de l'exploitation forestière. Elle décrypte les situations concrètes des différends et des conflits engendrés par les dispositifs techniques mis en œuvre, censés produire une meilleure gouvernance locale à propos des ressources. Ces dispositifs se traduisent par des modalités nouvelles de discrimination concrétisées, entre autres, par des prélèvements de taxes sur chaque sac de charbon de bois produit par des exploitants " étrangers ». La notion d'étranger perd ainsi de son élasticité à mesure que les conflits s'accentuent, se réduit aux origines géographiques et ethniques dont on sait pourtant le caractère construit et relatif (Amselle 1990, 1999, Amselle et M'Bokolo 1985, Poutignat et Streiff-Fenart, 1995). Revalorisation relative des traditions, des savoirs locaux, des ethnies, des castes et des métiers de charbonniers, de bûcherons, mais aussi prise en compte des incidences du conflit ivoirien voisin, des crises économiques, des crises politiques : tous ces éléments concourent à augmenter la valeur économique d'une activité jusque là dévalorisée et durcissent les stéréotypes autochtones / allochtones. Le bûcheronnage devient même l'activité principale d'un certain nombre de villageois au même titre que l'agriculture. La rareté de la ressource contribue au renchérissement du prix du sac de charbon et l'intérêt pour l'exploitation forestière malgré la pénibilité de la tâche et le mépris dont elle est l'objet.

Si la décentralisation et la réorganisation locale dans la gestion des ressources forestières au Mali réinventent la question d'autochtonie, on peut également le comprendre dans le texte de Seydou Keïta, à travers la réappropriation de la " tradition " par les associations de chasseurs, nouveaux gardiens des espaces forestiers. Une tradition quelque peu folklorisée dans la veine culturaliste qu'inspirent aussi les approches normatives des modèles d'économie institutionnelle véhiculés par les projets de développement. Pourtant, l'autochtonie est moins à lire dans l'acception du culturalisme que comme une conséquence des programmes mis en œuvre dans une vision libérale de la gestion rationnelle des ressources naturelles. 
Le rapport autochtone -allochtone apparaît aussi au Niger dans l'article d'Abdoulaye et Ibrahima. Outre cette dimension, ces auteurs montrent également le caractère pragmatique au quotidien des réformes « $d$ 'en bas». Les projets de développement y sont comme autant d'outils de légitimation politique. Dès lors, les acteurs locaux, élus et représentants des populations, s'autorisent à aller au-delà du seul rôle d'arbitre ou de justicier pour réguler les tensions du multi-institutionnalisme produit par les réformes "d'en haut» dans les enjeux de l'appropriation des ressources naturelles et des terres.

Ces exemples montrent que l'éviction du politique - au sens noble du terme, c'est à dire le politique comme mode d'action pour la gestion de la chose publique - est au cœur des projets de développement et qu'elle tend à réduire les conflits d'intérêts sur la ressource dans l'espace-temps des guerres picrocholines entre usagers, mobilisant le registre de l'autochtonie, alors même que ces guerres sont souvent le produit direct des dispositifs mis en œuvre. A partir de ces conflits au sein des institutions locales et des comités ad hoc, nous interrogeons les modèles développementalistes qui mobilisent les concepts de l'économie libérale des institutions internationales.

\section{La dimension technocratique et performative des projets de développement et de leur discours}

Le discours développementaliste sur la gestion des ressources naturelles, comme nous le rappelle Philippe Lavigne Delville (2005), est ambigu à plus d'un titre. Entre la "gestion déconcentrée participative » et la "gestion décentralisée ", il autorise les pratiques techniciennes et gestionnaires dont l'efficacité tant écologique que sociale ou économique n'est pas prouvée jusque là (cf. texte de Boutinot dans ce numéro).

On peut questionner le rôle des projets, tels ceux de la Banque Mondiale, à travers la SED (Stratégie Energie Domestique au Niger, Mali et Sénégal), dans la valorisation de l'exploitation forestière alors même que leur objectif était de la rationnaliser, voire de la remplacer par d'autres ressources énergétiques alternatives. Au-delà des processus de délégation de pouvoir aux collectivités locales, la mise en œuvre de tels projets de gestion des forêts "libéralise " encore davantage un secteur d'activités 
dont la spécificité est pourtant d'avoir les caractéristiques d'un bien commun. Des dispositifs qui, au lieu d'articuler action collective locale et action publique, participation locale et représentation politique dans le cadre de la décentralisation, tendent à instrumentaliser les processus de gestion locale au profit des logiques propres de l'aide (Lavigne Delville, $2005: 6$ ). On ne voit jamais aussi bien ces stratégies instrumentales que lorsque se confronte, notamment au Sénégal, la logique de l'appui budgétaire face aux projets de développement de grande ampleur comme ceux que la Banque mondiale finance et qui passent outre les instances parlementaires nationales et contrarient parfois même les réformes des ministères directement concernés (voir les articles de Boutinot et de Blundo).

Le développement de la filière du charbon de bois à partir de, et participant à, la concurrence entre les exploitants fait apparaître au Mali un système qui concentre petit à petit le pouvoir entre les mains de quelques uns. Alors qu'au Sénégal le même projet de gestion des ressources forestières était implicitement importé pour précisément mettre à l'épreuve un oligopole jugé trop puissant. Dans ces deux cas, les élus locaux et les autorités coutumières subissent plus qu'elles ne contrôlent les contradictions des réformes politiques, entre les approches sectorielles et les approches territoriales. En revanche, l'exemple du Niger, qui traite de ressources naturelles moins spéculatives, nous montre le pragmatisme avec lequel les acteurs de la décentralisation, élus locaux et autorités coutumières, utilisent les ressources et l'image des projets de développement comme discours et légitimité de la pratique politicienne.

La dialectique des réformes -du point de vue d'en haut et du point de vue d'en bas- est ainsi bien visible dans tous les articles de ce dossier. On peut le voir à travers les données empiriques ainsi que dans la description de la méthode (studying down, up and throught) que présente Giorgio Blundo. Ces études de cas illustrent les logiques d'action et les pratiques, de même que le rapport de ces pratiques aux discours et aux concepts de l'économie institutionnelle qui forment les cadres opérationnels des projets de développement dans la gestion des ressources forestières. 


\section{Les limites des cadres conceptuels}

de la «bonne gouvernance » : vers un dialogue entre l'économie institutionnelle et la socio-anthropologie?

D’une manière inédite, ce dossier de la Revue Anthropologie \& développement propose une rencontre avec l'économie institutionnelle (et néoinstitutionnelle). Cet exercice suggère une certaine familiarité avec les concepts qui sont mobilisés dans les projets de gestion des ressources communes (Community based natural resource management - CBNRM) mis en œuvre par les institutions internationales, la Banque mondiale et les organisations non gouvernementales depuis une vingtaine d'années. Ces concepts ou hypothèses des économistes (coûts de transaction, asymétrie de l'information, transparence, redevabilité) utilisés dans les discours et dans l'application des objectifs de la " bonne gouvernance ", sont ici questionnés.

Si les changements institutionnels et les réformes des politiques de gestion des ressources naturelles sont nécessaires pour réguler les rapports entre les services de l'Etat et les usagers, les codes, chartes ou contrats de gestion se sont imposés dans un double objectif. D'une part, celui de pallier les désengagements de l'Etat et, d'autre part, de composer avec des modes coutumiers dont la légitimité locale, fragilisée par les interventions politiques depuis l'époque coloniale, puis des indépendances (Lavigne Delville, $2006: 158$ ), est l'enjeu, selon les cas, d'une nouvelle reconnaissance ou d'une remise en question. En effet, les diverses formes de convoitises, d'exploitation ou d'accaparement dont font l'objet les espaces et les ressources naturelles, du fait des contraintes politiques et économiques, donnent lieu à une pluralité d'acteurs et d'usages et, partant, à une multiplications des normes et un nouveau partage des responsabilités. Mais malgré leur mise en œuvre depuis plus d'une décennie, ces chartes ou contrats de gestion sont difficilement suivis et respectés (Benkahla et Hochet, 2013), car ils nécessitent des négociations et la prise au sérieux des contextes socio-politiques. Or, dans la conception quelque peu abstraite et standardisée de la " bonne gouvernance ", il est rarement fait cas des contextes sociaux et politiques spécifiques. Le texte d'Antona, Boutinot et Kassibo fait l'hypothèse que ces contrats de gestion des ressources naturelles connaissent des échecs et sont parfois même des sources de conflits dans la gouvernance locale, en raison de cette conception techni- 
ciste, ainsi que de ses présupposés issus de l'économie néo-institutionnelle et institutionnelle dont s'inspirent les principaux bailleurs de fonds pour leur mise en œuvre (Olivier de Sardan, 2013). Dans les études de cas présentées ici, nous voyons réapparaître ces contextes en filigrane, souvent oubliés dans les approches normatives de la "bonne gouvernance ". Ils nous donnent à comprendre les limites des cadres conceptuels des économistes : négocier pour réduire les coûts de transactions, participer pour réduire les asymétries d'informations, favoriser l'empowerment pour contrôler l'accountability des dirigeants, etc.

Comme nous le rappelle Olivier de Sardan (2013), l'économie institutionnelle, nouvelle et ancienne, est entrée par les normes pour sortir l'approche économiste de la seule échelle individualiste. Cependant, elle ne permet pas davantage la compréhension des ressorts de l'action collective dès lors qu'elle continue à évacuer à la marge, et dans une vision culturaliste ce qu'elle nomme les comportements habituels ou les traditions culturelles, des réalités qui, de fait, sont le produit de l'encastrement des logiques sociales et politiques à l'œuvre dans les enjeux de la régulation sur les ressources naturelles et des territoires.

L'économie institutionnelle et la nouvelle économie Institutionnelle (NEI) ont l'intérêt d'ouvrir l'économie aux autres sciences sociales telles que l'anthropologie. Mais elles pêchent encore par leur manque de données empiriques (Olivier de Sardan, 2013) notamment pour rendre compte des formes de l'embeddedness. Le texte d'Antona, Boutinot et Kassibo illustre ces glissements de paradigme au sein du monde économique, ouvrant la porte aux apports anthropologiques. Il est ici question du rapport à ces courants dominants de l'économie qui saturent les politiques publiques, et de leur mise en œuvre dans les programmes et projets de développement; rapport au sein duquel l'anthropologie a maille à partir. L'organisation de la gouvernance à partir de la multiplication des institutions locales, avec comme présupposé que celles ci seront susceptibles, à force de négociation et à l'aide d'incitations (notamment économiques), de partager les mêmes intérêts sur le maintien durable d'une ressource, renvoie à une vision technocratique du développement. Les chartes locales ou les contrats de gestion y sont des mises en forme (et mises " avec des formes ", pour reprendre la formule de Pierre Bourdieu) de l'espace de production et des rapports sociaux. Présentés, en effet, comme des ins- 
truments performatifs de création du consensus et de conciliation, ces contrats de gestion viennent en réalité contrevenir aussi bien aux modes coutumiers de l'exercice de l'autorité sur les territoires, qu'aux tentatives récentes de démocratisation de l'espace public et des réformes politiques de la décentralisation.

La comparaison de la mise en œuvre de ces outils de gestion que sont les contrats et chartes locales, entre le Niger, le Mali et le Sénégal, montre une certaine standardisation des réformes d'en haut et des réponses et micro réformes d'en bas originales. L'analyse de l'économie institutionnelle rend compte et participe de la forme standardisée des dispositifs mais le recours à l'anthropologie du développement reste nécessaire à la compréhension des pratiques locales, au-delà des normes, dans le sens que leur donnent les acteurs concernés. Ce dont l'ensemble des articles de ce dossier témoigne.

\section{«Réformes par le haut et réformes par le bas »}

Il est certain que décentraliser la compétence politique sur la gestion foncière et sur celle des ressources naturelles n'était pas moins risqué dans ces trois pays. Les irrédentismes historiques des régions et des populations longtemps écartées des avantages du développement demandaient aux gouvernants du courage (ou une prise de risque imposée par le modèle libéral) pour accepter des formes d'autonomie politique locale sur le territoire. Comme nous le rappelait Bréhima Kassibo (1997 : 17), les réformes territoriales et les politiques de décentralisation au Mali comportaient un défi démocratique majeur qui consistait à élargir l'espace de l'exercice du pouvoir à un plus grand nombre de citoyens, en vertu des compétences et non d'un rang de naissance et de privilèges. Mais dans le processus de décentralisation qui vient se superposer à ce qui reste des institutions précoloniales et coloniales - ici le choix des chefs-lieux de communes qui ont été autrefois le théâtre d'affrontements au Mali, là des relents de l'autorité administrative coloniale où le maire est perçu comme l'ancien " baba coumantan " (Kassibo, 1997) - il existe des ressentiments, des peurs et des craintes qui, partout, révèlent l'hétérogénéité du monde rural. Et si les dispositifs de gouvernance locale pour la gestion des ressources naturelles réactivent d'anciens conflits, on le voit ici encore à tra- 
vers la délimitation d'un territoire qui se traduit en découpages " d'en haut » à la mode coloniale (Sénégal) ou bien "d'en bas » pour la mise en œuvre de plan d'aménagement ou de plan d'approvisionnement des villes en bois énergie (Mali, Niger). Souvent ces découpages occultent "l'importance du vote pour le choix démocratique des élus, (...) dans un souci de rééquilibrage des pouvoirs au sein de la collectivité décentralisée " (Kassibo, 1997). A travers la question du bois énergie et du charbon de bois, ces exemples donnent à voir le biais par lequel les réformes venant d'en haut peuvent être court-circuitées en bas, certes, ... mais aussi par le haut ! Comprenne qui pourra. Les enchevêtrements d'intérêts et le multi institutionnalisme qui s'y développe, tendent à détruire les valeurs de la redevabilité et à confondre les responsabilités à toutes les échelles d'autorité.

Le texte de Boutinot montre ce que produit la contradiction des réformes dès lors qu'il est question de l'appropriation des espaces et des ressources nécessaires à une production dont la valeur ajoutée est hautement lucrative et détenue par des entrepreneurs puissants. Une brèche entre des dispositifs technocratiques et la dynamique des pratiques locales favorise l'invention d'alternatives, ici, dans une lutte serrée mais tout de même encore au profit de ceux qui craignent qu'une décentralisation des compétences sur la gestion des territoires et des ressources soit véritablement ce que Bréhima Kassibo appelait le lieu possible d'un partage équitable des biens et des richesses du pays.

II arrive que la formalisation par le haut des contrats de gestion augmente les coûts de transactions au lieu de les réduire, comme on peut le lire dans le texte d'Antona, Boutinot et Kassibo. C'est alors que ces contrats deviennent autant de lieux stratégiques de captation de la rente du développement apportée par les bailleurs internationaux et les Organisations non gouvernementales. Cependant que certaines formalisations, par le bas, autorisent une redistribution des ressources autant légales qu'illicites au sein des services forestiers, à la fois individuellement, mais aussi au profit du maintien du service forestier lui-même, et c'est surtout cela qui est éloquent dans le texte de Giorgio Blundo. Je cite : "la corruption dans certains cas peut assurer la survie d'un service public ", bien démuni au Sénégal, comme partout ailleurs en Afrique de l'Ouest, depuis les politiques d'ajustement structurel. Ironie? Boutinot montre que le 
projet de la Banque mondiale avait, parmi ses objectifs, celui de "libéraliser » la filière de charbon de bois et de soutenir la concurrence des petits producteurs pour démanteler la structure oligopolistique. Les malversations décrites par G Blundo au sein de ce projet viennent affaiblir la notoriété de ce dispositif et sa légitimité à poursuivre ses objectifs. Il est certain qu'à multiplier les instances de décisions au niveau micro local ainsi que ce projet l'a fait dans la création de multiples Comités Villageois et Inter Villageois de gestion, il se produit un enchevêtrement des intérêts dont la conséquence est de diluer et d'affaiblir les responsabilités et partant, de favoriser les pratiques corruptives (Polanyi, [1944] 1983 : 169).

Par ailleurs, l'article de Blundo présente une administration forestière méfiante envers les collectivités locales et qui les accuse de tous les maux : elles monnayeraient les autorisations de coupes, seraient insensibles aux questions environnementales, etc. Le texte de Boutinot ne montre d'ailleurs pas l'inverse, mais nuance le propos puisque si les services forestiers traditionnels n'ont pas l'air de faire grand cas des représentants des collectivités locales élues, c'est parce qu'ils préfèrent travailler comme à leur habitude à l'échelle des villages et des chefs de villages lorsqu'il s'agit d'installer les exploitants forestiers en brousse. Ces visions ne manquent pas non plus de souligner les contradictions au sein du service forestier entre sa mission de gestion de l'environnement, participative, qui exige des résultats visibles et les diverses pratiques moins visibles, les "relations collusives entre agents forestiers, producteurs et usagers de l'administration " (Blundo) qui contreviennent aux projets d'aménagement forestier et à la logique de fiscalisation de la filière de charbon de bois.

Mais l'ethnographie de l'éthique professionnelle du corps forestier montre finement les paradoxes dans lesquels sont pris ces agents forestiers partagés entre militarisation et participation (Blundo). Ce qui nous fait dire que nous poursuivons les analyses empiriquement documentées de la "dimension publique de la politique " (Olivier de Sardan, 2009 : 16), celle de l'Etat et de ses agents et de ses appareils qui ne sont pas seulement des dispositifs de répression mais aussi des dispositifs de fonctionnement plus ou moins efficaces qui assurent "protection, assistance, fourniture de services, développement et création d'infrastructures " (Olivier de Sardan, op cit: 17). Les services forestiers, dont on voit qu'ils ne sont pas un groupe homogène représentant l'Etat dans sa toute puissance, sont en 
réalité traversés par des tensions et des divisions. Ils présentent des désaccords que révèle la difficile conjugaison de la réforme nationale de l'institution avec les projets de développement forestiers des bailleurs de fonds.

Au-delà des pratiques corruptives et de détournement de fonds au profit d'intérêts personnels et privés dont les projets de développement ne sont pas plus exempts que les administrations publiques, le cas sénégalais de la gestion des forêts illustre ce que Giorgio Blundo appelle à la fois la nouvelle mission assignée au service forestier dans le cadre de la réforme de l'institution: "une militarisation [et] le renforcement de l'alliance avec les exploitants contre les élus et les populations locales » et les réactions divergentes de nombre d'agents forestiers eux-mêmes face à cette mission. Les deux réformes simultanées de l'institution forestière se présentent telle "la figure de l'oxymore en politique publique ". D'un côté, la militarisation qui confère le prestige à ses agents dans le port de l'uniforme et permet d'accroître les salaires et l'autorité policière ; d'un autre côté, l'animation rurale et le soutien à l'approche participative de la gestion décentralisée des espaces forestiers, suscitant, à l'inverse, quelques découragements.

Enfin, le travail de recherche avec les acteurs qui sont représentants d'un corps militaire et sensibles au prestige que l'uniforme nouvellement reconquis symbolise, mais aussi avec les gens ordinaires et avec les représentants des nouvelles autorités élues, tous aux prises avec les ambiguïtés, les oppositions et les conflits de la vie publique, et qui, bien souvent, maîtrisent les règles du jeu de l'enquête, mobilise les postures compréhensive et réflexive du chercheur. Notre cher ami et collègue disparu, Bréhima Kassibo, insistait sur cette rigueur nécessaire à une production scientifique qui rende compte de la réflexivité des agents eux-mêmes, des animateurs de projets dans leurs pratiques comme des ministres dans leurs prises de décisions politiques; réflexivité qu'il n'avait de cesse de s'appliquer à luimême et qu'il enseignait à ses étudiants avec fermeté et bienveillance.

Que la terre te soit légère, Bréhima. 


\section{Bibliographie}

AMSELLE, J-L., [1990] 1999, Logiques métisses: anthropologie de l'identité en Afrique et ailleurs, Paris, Payot 257p.

AMSELLE, J-L. et M'BOKOLO, E., (dir.), 1985, Au cœur de l'ethnie: ethnies, tribalisme et État en Afrique, Paris, La Découverte, 227p.

BAKO-ARIFARI N et J-P LAURENT, 1998, (ss.dir.), Les dimensions sociales et économique du développement local et la décentralisation en Afrique au Sud du Sahara, Bulletin de l'APAD, N¹5.

BIERSCHENCK, T., 2010, Historiciser et localiser les approches, Anthropologie et développement, Bulletin de l'APAD, $\mathrm{n}^{\circ}$ 31-32 : 161-192.

BLUNDO, G. et R. MONGBO (Eds.),1998, Décentralisation, pouvoirs locaux et réseaux sociaux, Bulletin de l'APAD, $\mathrm{N}^{\circ} 16$.

BOUTINOT, L., DIOUF, C.N., 2006, "Quand certaines approches participatives engendrent des formes ambiguës de mobilisation de la société civile. Quelques exemples à propos de la gestion des ressources naturelles au Sénégal », In : BERTRAND, A., KARSENTY, A. et MONTAGNE, P. (eds.), L'Etat et la gestion locale durable des forêts en Afrique francophone et à Madagascar, Paris, L'Harmattan,pp. 192 -208.

KASSIBO B.(Ed.), 1997, La décentralisation au Mali : état des lieux, Bulletin de I'APAD N 14.

LAVIGNE DELVILLE, P., 2006, Conditions pour une gestion décentralisée des ressources naturelles. Entre " community failures », " market failures » et "state failures", construire de nouveaux communs, In : BERTRAND, A., KARSENTY, A. et MONTAGNE, P. (eds.), L'Etat et la gestion locale durable des forêts en Afrique francophone et à Madagascar, Paris, L'Harmattan,pp. 153-173.

LAVIGNE DELVILLE, P., HOCHET, P., 2005,Construire une gestion négociée et durable des ressources naturelles renouvelables en Afrique de l'Ouest, Rapport final de l'étude AFD/UE, Paris, GRET, $180 \mathrm{p}$.

Le MEUR, P-Y. et C. LUND, (ss.dir.), 2001, Gouvernance foncière au quotidien en Afrique, Bulletin de l'APAD $\mathrm{N}^{\circ} \mathrm{s} 22$ et Gestion des ressources naturelles: participations et médiations,2003, Bulletin de l'APAD $\mathrm{N}^{\circ} 26$.

OLIVIER DE SARDAN, J-P, 2009, «Gouvernance chefferiale et gouvernance associative dans les campagnes nigériennes $»$, In 
OLIVIER DE SARDAN J-P., 2013, Embeddedness and informal norms : institutionalisms and anthropology, Critique of Anthropology, 33 (3): 280299.

POUTIGNAT, P., et STREIFF-FENART,J., 1995, Les théories de l'ethnicité, Paris, PUF, $272 p$.

POLANYI, K., 1983, La grande transformation aux origines de notre époque, Paris, Gallimard, 1983 (ed. anglaise, 1944), 420p.

RIBOT, J., PELUSO NL., 2003, A theory of acces, Rural Sociology, Vol. 6, n²: 153181.

\section{Aviviviv}

Laurence Boutinot est socio-anthropologue au Cirad.

E-mail : laurence.boutinot@cirad.fr 